\title{
Empleo de DSSAT-PNUTGRO en la región central de Argentina: evaluaciones de fechas de siembra con información histórica y escenarios de cambio climático
}

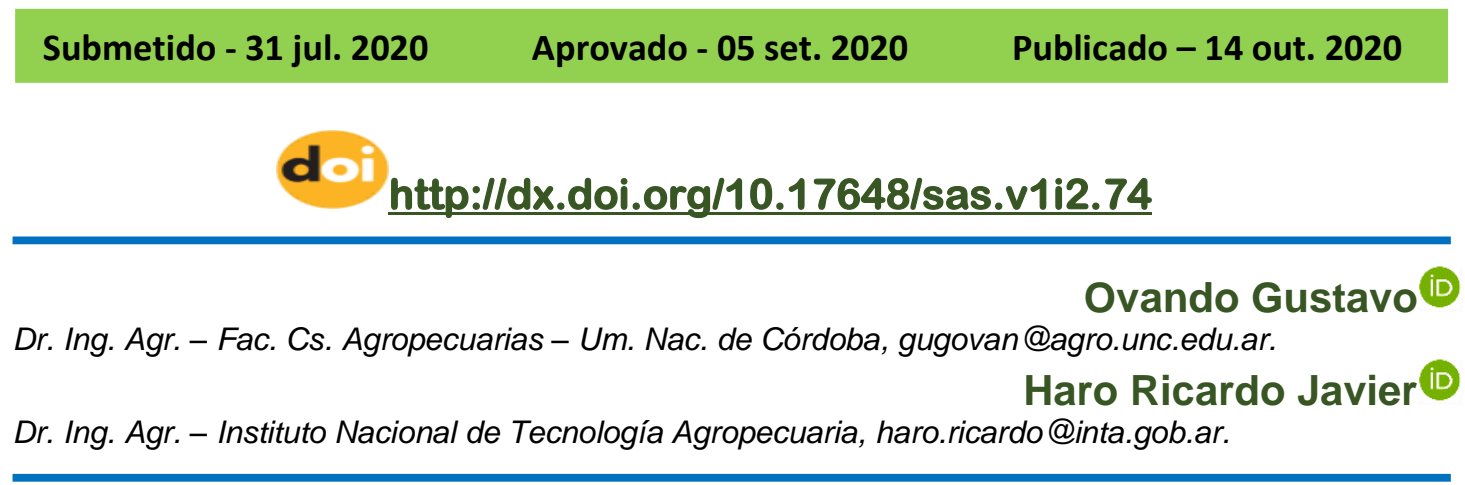

\section{RESUMEN}

El cultivo de maní es importante en la región central de Argentina y se realiza principalmente en secano, por lo que resulta importante establecer una fecha de siembra adecuada para que el cultivo aproveche las precipitaciones durante su ciclo de cultivo. La fecha de siembra podría incluso ser modificada debido a los efectos del cambio climático. Los modelos de cultivos permiten evaluar los efectos del clima, el suelo, el manejo sobre el rendimiento del cultivo y su variabilidad. Los objetivos de este trabajo consistieron en emplear el modelo Decision Support System for Agrotechnology Transfer (DSSAT) para identificar una fecha de siembra óptima utilizando datos climáticos históricos y determinar los efectos del cambio climático en el rendimiento de maní. A tal efecto, se realizaron simulaciones para el cultivar ASEM 400 INTA con información meteorológica histórica (1973-2019) y de un futuro cercano (2030-2064) y lejano (2065-2099) con cuatro escenarios Representative Concentration Pathways (RCP: $2.6 ; 4.5$ 6.0 y $8.5 \mathrm{Wm}^{-2}$ ), combinando tres fechas de siembra y tres condiciones de contenido de agua en el suelo al momento de la siembra. Las simulaciones realizadas con información meteorológica histórica estiman mayores rendimientos con fechas de siembras más tempranas y la variabilidad de esos rendimientos disminuye cuanto mayor sea el porcentaje de agua útil al momento de la siembra. También se observa una disminución de la variabilidad con mayor contenido de agua a la siembra para condiciones futuras para todos los escenarios analizados. En la mayoría de las condiciones futuras y escenarios los rendimientos medianos se incrementan, en relación a sus respectivos tratamientos con información meteorológica histórica. El mayor incremento mediano de rendimiento de maní se obtiene en un futuro lejano con un RCP de $8.5 \mathrm{Wm}^{-2}$ y $70 \%$ de agua útil a la siembra.

Palavras-chave: Arachis hypogaea; Distribuição geográfica; Thecaphora frezzii.

\section{Use of the DSSAT-PNUTGRO in Argentina's central region: evaluations of sowing dates using historical data and climate change scenarios}

\begin{abstract}
The peanut is an important crop in Argentina's central region and it is mainly sown in dry land. Thus, it is important to establish an appropriate planting date to take advantage of rainfall during its growth cycle. This date could be even modified due to climate change effects. Crop models allow evaluating the effects of climate, soil and management on crop yield and its variability. The goal of this work was to use the Decision Support System for Agrotechnology Transfer (DSSAT) model to identify an optimal sowing date using historical climate data and determine the climate change effects on peanut yield. To this end, simulations were performed for the ASEM 400 INTA cultivar using historical weather data (1973-2019) and, from the near future (2030-2064) and the distant future (2065-2099) with four Representative Concentration Pathways scenarios (RCP: 2.6, 4.5, 6.0 and $8.5 \mathrm{Wm}^{-2}$ ), combining three sowing dates and three
\end{abstract}


conditions of soil water availability at the sowing date. Simulations performed with historical climate data estimate higher yields in earlier sowing dates and, the variability of those yields decrease when there is higher water availability at the sowing date. A decrease in variability with higher soil water content at the sowing date is also observed for future conditions in all the scenarios analyzed. In most future conditions and scenarios, the medium yields increase respect to their treatments with historical weather data. The greatest median increase in peanut yield is obtained in the distant future with a RCP of $8.5 \mathrm{Wm}^{-2}$ and $70 \%$ of water availability at the sowing date.

Keywords: Droughts; RCP; distant future.

\title{
Uso do DSSAT-PNUTGRO na região central da Argentina: avaliações de datas de semeadura com informações históricas e cenários de mudanças climáticas
}

\author{
RESUMO
}

O cultivo de amendoim é importante na região central da Argentina e é realizado principalmente na época da seca, por isso é importante estabelecer uma data ideal de semeadura para que aproveite as chuvas durante seu ciclo de cultivo. Alémdisso, a data de semeadura pode ser modificada pelos efeitos das mudanças climáticas. Os modelos de culturas permitem avaliar os efeitos do clima, solo, manejo no rendimento das culturas e sua variabilidade. Objetivamos identificar a data ideal de semeadura com 0 modelo Decision Support System for Agrotechnology Transfer (DSSAT), utilizando dados climáticos históricos e determinar os efeitos das mudanças climáticas no rendimento do amendoim. Para isso, simulações foram realizadas com a cultivar ASEM 400 INTA, por meio de informações meteorológicas históricas (1973-2019), no futuro próximo (2030-2064) e no futuro distante (2065-2099) com quatro cenários de Representative Concentration Pathways (RCP: 2,6; 4,5 6,0 e 8,5 W $\mathrm{m}^{-2}$ ), combinando três datas de semeadura e três condições de teor de água no solo no momento de semeadura (30, 50 e $70 \%$ de água útil). Simulações com informações meteorológicas históricas estimaram rendimentos mais altos em datas de plantio anteriores e, esses rendimentos aumentaram e sua variabilidade foi menor com maior quantidade de água útil na semeadura. Para os cenários analisados, também foi observada diminuição na variabilidade com maior teor de água no plantio para condições futuras. Por meio das informações meteorológicas históricas, na maioria das condições e cenários futuros, os rendimentos médios aumentaram em relação aos seus respectivos tratamentos. O maior aumento médio na produção de amendoim foi obtido em um futuro distante com RCP de $8,5 \mathrm{Wm}^{-2}$ e $70 \%$ de água útil no plantio.

Palavras-chave: Secas; RCP; futuro distante.

\section{Introdução}

El maní (Arachis hypogaea L.) se cultiva en la región central de Argentina, ocupando unas 316000 ha, con un rendimiento promedio de 3.3 t ha-1. La producción de maní en la provincia de Córdoba se realiza en secano, con precipitaciones dentro de la estación de crecimiento de 600 $\mathrm{mm}$. Existe una considerable variabilidad en la cantidad y distribución de las precipitaciones en la provincia, tanto dentro como entre las estaciones de cultivo, llevando a sequías que varían en duración y severidad, reduciendo de esa manera el rendimiento del cultivo por debajo de los 
esperados. Las prácticas actuales de manejo involucran pocos cultivares, una ventana temporal de siembra durante la primera mitad de noviembre, espaciamiento entre surcos de $0.7 \mathrm{~m}$ y densidad de siembra de 14 plantas $\mathrm{m}^{-2}$.

La siembra generalmente se lleva a cabo durante la primera mitad de noviembre, pero es frecuentemente extendida hasta mediados y fines de diciembre. La fecha de siembra óptima puede determinarse a través de ensayos a campo en varios años, un método alternativo es emplear un modelo de cultivo junto con información climática histórica. La fecha de siembra óptima podría ser modificada en el futuro debido a los efectos del cambio climático que influirían directamente en la distribución espacial y temporal de los elementos básicos de la producción agrícola (e.g. radiación solar, agua y temperatura) (TUBIELLO et al., 2007; TAN et al., 2013), como también sobre eventos meteorológicos extremos y variabilidad climática.

Los modelos de cultivo se utilizan para evaluar los efectos del clima, el suelo, el manejo y otros factores sobre el rendimiento del cultivo y su variabilidad. Al comparar las estrategias de gestión de cultivos con el clima actual y esperado, esos modelos también ayudan a los agricultores a reducir los riesgos de producción y aumentar el rendimiento de los cultivos (BOOTE et al., 2011; YADAV et al., 2012). En este sentido, el modelo DSSAT-PNUTGRO se utilizó para evaluar los impactos del cambio climático en la producción de maní en China (XU et al., 2017), en el rendimiento del maní en Senegal utilizando cuatro modelos climáticos y dos escenarios de cambio climático (SARR y CAMARA, 2018). Faye y colaboradores (2018) analizaron con modelos de simulación de cultivos, el efecto de dos escenarios de cambio climático sobre el rendimiento de maní en el oeste de África.

Nuestros objetivos son (i) identificar una fecha de siembra óptima utilizando datos climáticos históricos con el modelo PNUTGRO (DSSAT V4.7) para la región central de Argentina, y (ii) determinar los efectos del cambio climático en el rendimiento de maní. 


\section{Materiales y Métodos}

Para las simulaciones con DSSAT se emplearon valores diarios de radiación solar, temperatura máxima y mínima y precipitaciones de los años 1973 a 2019 (considerados como datos históricos) de la estación experimental Manfredi ( $31^{\circ} 24^{\prime} \mathrm{S}, 61^{\circ} 11^{\prime} \mathrm{W}, 297 \mathrm{msnm}$ ), perteneciente al Instituto Nacional de Tecnología Agropecuaria (INTA). El suelo de la zona pertenece a un Haplustol típico con $150 \mathrm{~mm}$ de agua disponible por metro de profundidad (DARDANELLI et al., 2003).

Para este estudio se emplearon los coeficientes genéticos, previamente calibrados, del cultivar ASEM 400 INTA (OVANDO y HARO, 2019). Este cultivar es del tipo runner, con un ciclo de cultivo corto, de aproximadamente 140 días y en la actualidad es sembrada en el área manisera de Argentina. La siembra se simuló en tres fechas contrastantes del 15-oct, 15-nov y 15-dic (en adelante serán mencionadas como fechas de siembra temprana, intermedia y tardía, respectivamente), respetando las condiciones de manejo de los productores, es decir con surcos espaciados a $0,7 \mathrm{~m}$ y una densidad de siembra de 14 plantas $\mathrm{m}^{-2}$. Cada fecha de siembra se simuló con un contenido de agua inicial del 30,50 y $70 \%$ del agua útil (AUS), considerando una profundidad del suelo de $2 \mathrm{~m}$.

Los datos meteorológicos futuros fueron estimados por un promedio de un conjunto de 17 Modelos Globales de Circulación disponibles en la aplicación MarkSim ${ }^{\mathrm{TM}}$ DSSAT Weather File Generator (JONES y THORNTON 2000). Se usaron dos conjuntos de datos diarios, el primero entre los años 2030-2064 y el segundo entre 2065-2099. Este conjunto multi modelos considera cuatro escenarios futuros conocidos como Representative Concentration Pathways (RCP); que es un conjunto de trayectorias de concentración de gases de efecto invernadero adoptada por la comunidad de modelado climático como base para el modelado de experimentos de corto a largo plazo. Las RCPs consideradas fueron 2.6, $4.5,6.0$ y $8.5 \mathrm{Wm}^{-2}$.

Los productos empleados en este estudio es un conjunto de datos con una resolución espacial de 30 segundos de arco derivadas de 
WorldClim (HIJMANS et al., 2005) para períodos de mediados (2030-2064) y finales del siglo XXI (2065-2099). En cada archivo meteorológico generado, se agregó el valor de la concentración de $\mathrm{CO}_{2}$ correspondiente a cada año y RCP, que fue obtenida a partir de determinaciones de Meinshausen et al. (2011).

\section{Resultados y Discusión}

Los resultados de las simulaciones se muestran en la Figura 1. En ella puede observarse que para las corridas del modelo con los datos históricos (1973-2019), los valores medianos del rendimiento del cultivo aumentan a medida de se adelanta la fecha de siembra y a medida que aumenta el contenido de agua útil al momento de la siembra, con valores extremos entre $1694 \mathrm{~kg} \mathrm{ha}^{-1}$ para la fecha de siembra tardía con $30 \%$ de AUS y $4405 \mathrm{~kg} \mathrm{ha}^{-1}$ para la fecha de siembra temprana con $70 \%$ de AUS. El retraso en la fecha de siembra produce una mayor velocidad de emergencia de las plantas, pero expone al cultivo a menores niveles de temperatura y radiación en el llenado de grano, con consecuentes disminuciones en el rendimiento. Por otra parte, los cultivos sembrados en fechas tempranas están expuestos a incrementos en los niveles de radiación y temperatura durante la fijación de vainas y llenado de grano, condiciones que resultan beneficiosas para el rendimiento. Sin embargo, las bajas temperaturas al comienzo del ciclo del cultivo pueden retrasar la germinación, lo cual pone en riesgo la emergencia de las plántulas (HARO et al., 2007).

El patrón de disminución de la variabilidad de los rendimientos que se observa con el incremento del porcentaje de agua útil al momento de la siembra se mantiene tanto para un futuro cercano (2030-2064), como para el futuro lejano (2065-2099), independientemente del valor de RCP.

La mayoría de los escenarios futuros presentan valores medianos superiores al tratamiento correspondiente simulado con datos históricos, lo cual sugiere que las combinaciones de cambios en la temperatura, precipitaciones y concentración de $\mathrm{CO} 2$, entre otros, permitiría un mejor desempeño del cultivar ASEM 400 INTA en la región manisera de Córdoba. 
Sólo las simulaciones con fecha de siembra de 15-oct y RCP de $4.5 \mathrm{Wm}^{-2}$ para un futuro cercano con un AUS de 30 y $70 \%$ produjeron diferencias negativas entre las medianas de 62.5 y $125 \mathrm{~kg} \mathrm{ha}^{-1}$, respectivamente. En tal sentido, Faye et al. (2018) demostraron que en condiciones de secano la concentración de $\mathrm{CO}_{2}$ presenta un efecto positivo en el rendimiento de semilla de maní cuando las temperaturas están por debajo de un umbral de $38^{\circ} \mathrm{C}$.
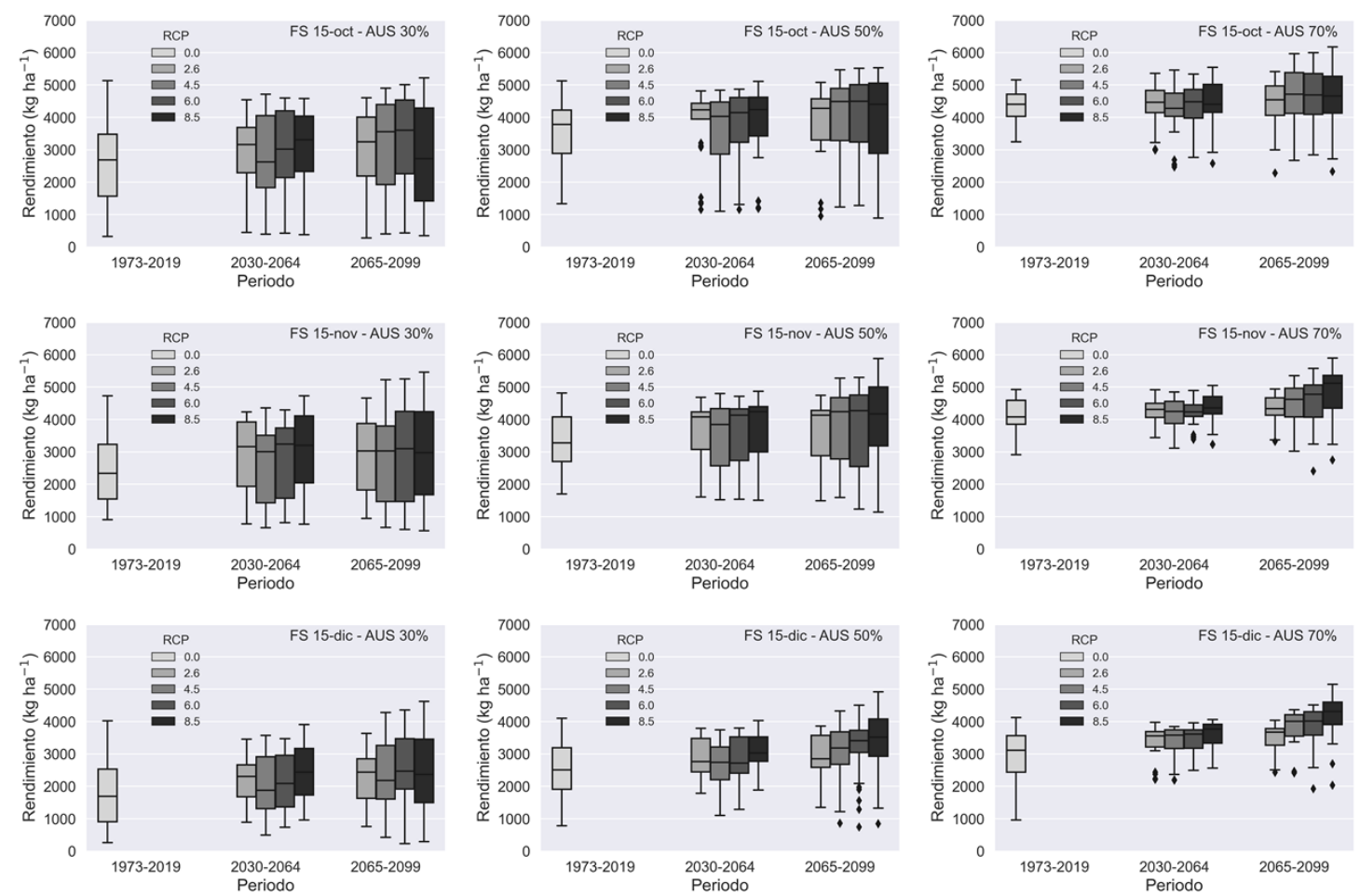

Figura 1. Gráficos de Boxplots de rendimientos de maní del cultivar ASEM 400 INTA para los tratamientos de fecha de siembra (FS: 15-oct; 15-nov y 15-dic) y contenido de agua útil a la siembra (AUS: 30\%; 50\% y 70\%), cada gráfico presenta los valores actuales y los valores futuros (2030-2064 y 2065-2099) para los escenarios correspondientes a los Representative Concentration Pathways de 2.6, $4.5,6.0$ y $8.5 \mathrm{Wm}^{-2}$.

Con respecto a las fechas de siembra y contenido de humedad al momento de la siembra, los mayores incrementos en los rendimientos medianos se expresan en promedio para las fechas del 15-nov con un contenido de agua útil al momento de la siembra de 30 y $50 \%$ e incrementos entre 564 y $966 \mathrm{~kg} \mathrm{ha}^{-1}$ para todos los RCPs.

Al analizar los distintos escenarios futuros, en general los incrementos medianos de rendimientos son mayores a medida que aumentan los valores de RCP (con excepción de $4.5 \mathrm{Wm}^{-2}$, para el futuro cercano) y tales aumentos 
son aún mayores en el futuro lejano (2065-2099). En consecuencia, el mayor incremento mediano ocurre para el futuro lejano con un RCP de $8.5 \mathrm{Wm}^{-2}$ con $70 \%$ de agua útil a la siembra (1191 $\left.\mathrm{kg} \mathrm{ha}^{-1}\right)$.

\section{Conclusiones}

Las simulaciones realizadas, con información meteorológica histórica, en la región central de Córdoba estiman mayores rendimientos en fechas de siembras más tempranas. A su vez, estos rendimientos se incrementan y su variabilidad disminuye cuanto mayor sea el porcentaje de agua útil a la siembra. También se observa una disminución de la variabilidad con mayor contenido de agua a la siembra para condiciones futuras para todos los escenarios analizados. En la mayoría de las condiciones futuras y escenarios, los rendimientos medianos aumentan respecto a sus respectivos tratamientos con información meteorológica histórica. El mayor incremento mediano de rendimiento de maní para el cultivar ASEM 400 INTA se obtiene en un futuro lejano ante un escenario Representative Concentration Pathways de $8.5 \mathrm{Wm}^{-2}$ con $70 \%$ de agua útil a la siembra. 


\section{Referências}

BOOTE, K. J.; IBRAHIM, A. M.; LAFITTE, R.; MCCULLEY, R.; MESSINA, C.; MURRAY, S.; SPECHT, J.E.; TAYLOR, S.; WESTGATE, M.E.; GLASENER, K.; BIJL, C.G.; GIESE, J.H. Position statement on crop adaptation to climate change. Crop Science, v.51, p. 2337-2343, 2011. https://doi.org/10.2135/cropsci2011.07.0369.

DARDANELLI, J.L.; CALMON, M.A.; JONES, J.W.; ANDRIANI, J.M.; DÍAZ, M.P.; COLLINO, D.J. Use of a crop model to evaluate soil impedance and root clumping effects on soil water extraction in three Argentine soils. Transactions of the American Society of Agricultural Engineers, v.46, p. 1265-1275, 2003. doi: 10.13031/2013.13946

FAYE, B.; WEBBER, H.; DIOP, M.; MBAYE, M. L.; OWUSU-SEKYERE, J. D.; NAAB, J. B.; GAISER, T. Potential impact of climate change on peanut yield in Senegal, West Africa. Field Crops Research, v.219, p.148-159, 2018. https://doi.org/10.1016/j.fcr.2018.01.034

HARO, R.J.; OTEGUI, M.E; COLLINO, D.J.; DARDANELLI, J.L. Environmental effects on seed yield determination of irrigated peanut crops: links with radiation use efficiency and crop growth rate. Field Crops Research, v.103, p. 217-228, 2007. https://doi.org/10.1016/j.fcr.2007.06.004

HIJMANS, R.J.; CAMERON, S.E.; PARRA, J.L.; JONES, P.G.; JARVIS, A. Very high resolution interpolated climate surfaces for global land areas. International Journal of Climatology, v. 25, p. 1965-1978, 2005. https://doi.org/10.1002/joc.1276

JONES, P.G.; THORNTON, P.K. MarkSim: software to generate daily weather data for Latin America and Africa. Agronomy Journal, v. 93, p. 445-453, 2000. https://doi.org/10.2134/agronj2000.923445x

MEINSHAUSEN, M.; SMITH, S. J.; CALVIN, K.; DANIEL, J. S.; KAINUMA, M. L. T.; LAMARQUE, J. F.; MATSUMOTO, K.; MONTZKA, S.A.; RAPER, S.C.B.; RIAHI, K.; THOMSON, A.; VELDERS, G. J. M.; VAN VUUREN, D.P.P. The RCP greenhouse gas concentrations and their extensions from 1765 to 2300. Climatic Change, v. 109, p. 213, 2011. https://doi.org/10.1007/s10584-011$\underline{0156-z}$

OVANDO, G.; HARO, R.J. Simulación de la fenología, crecimiento y rendimiento sin limitaciones hídricas de la variedad ASEM 400 INTA de maní con DSSAT Crop Peanut Model. XVI Encontro Sobre a Cultura do Amendoim 15 a 17 de agosto de 2019 na Estação de Eventos Cora Coralina e FCAV/UNESP, Jaboticabal-SP.

SARR, A. B.; CAMARA, M. Simulation of the impact of climate change on peanut yield in Senegal. International Journal of Physical Sciences, v. 13, p. 79-89, 2018. https://doi.org/10.5897//JPS2017.4710 
TAN, Z. H.; TANG, H. J.; LI, W. J.; ZHAO, S. H. Progress and directions in studying the impacts of climate change on agriculture and grain production in China. Chinese Journal of Agricultural Resources and Regional Planting, v.34, p. 1-7, 2013.

TUBIELLO, F. N.; SOUSSANA, J. F.; HOWDEN, S. M. Crop and pasture response to climate change. Proceedings of the National Academy of $\begin{array}{lllll}\text { Sciences, } & \text { v.104, } & \text { p. } & 19686-19690, & \end{array}$ https://doi.org/10.1073/pnas.0701728104

XU, H.; TIAN, Z.; ZHONG, H.; FAN, D.; SHI, R.; NIU, Y.; HE, X.; CHEN, M. Impacts of climate change on peanut yield in China simulated by CMIP5 multimodel ensemble projections. In: Remote Sensing and Modeling of Ecosystems for Sustainability XIV (Vol. 10405, p. 104050W). International Society for Optics and Photonics, 2017.

YADAV, S.B.; PATEL, H.R.; PATEL, G.G.; LUNAGARIA, M.M.; KARANDE, B.I.; SHAH, A.V.; PANDEY, V. Calibration and validation of PNUTGRO (DSSAT v4.5) model for yield and yield attributing characters of kharif groundnut cultivars in middle Gujarat region. Journal of Agrometeorology, v. 14, p. 24-29, 2012. 